\title{
Basilar artery ectasia: an unusual cause of trigeminal
} neuralgia

\author{
RICHARD H LYE \\ From University Department of Neurosurgery, Manchester Royal Infirmary, Manchester, UK
}

SUMMARY Basilar artery ectasia may be associated with trigeminal neuralgia as a consequence of trigeminal nerve compression by the aberrant artery. The case histories are presented of four patients, investigation of whom demonstrated the presence of an ectatic basilar artery thought to be the cause of facial pain. The value of intravenous digital subtraction angiography in the investigation of this condition is emphasised. The surgical management is discussed with particular reference to the choice between percutaneous thermocoagulation and posterior fossa microvascular decompression.

Posterior fossa exploration reveals a vascular anomaly of the pontine trigeminal root entry zone in a significant proportion of patients suffering from trigeminal neuralgia. ${ }^{1-3}$ The supposition that compression of the trigeminal nerve is caused by aberrant arterial or venous loops ${ }^{2}$ has been questioned, since such vascular anomalies are known to occur in patients reportedly not suffering from trigeminal neuralgia ${ }^{4}$ However, mobilisation of a vessel apparently compressing the trigeminal nerve followed by interposition of a prosthesis between the vessel and the nerve relieves the symptoms. ${ }^{2356}$ Basilar artery ectasia is an unusual cause of trigeminal neuralgia $^{1-8}$ secondary to vascular compression. The case histories of four patients suffering from this vascular anomaly are reviewed and the management of the patients discussed.

\section{Case reports}

Case 1

A 67-year-old Caucasian female presented with a 3 year history of trigeminal neuralgia. Her pain failed to respond to carbamazepine or diphenylhydantoin and she was therefore referred to the neurosurgical department. On examination, she was generally fit. Neurological examination revealed minimal hypalgesia and hypaesthesia in the first division of her right trigeminal nerve territory. Her pain was in the second trigeminal division on the right with a

Address for reprint requests: Mr RH Lye, University Dept of Neurosurgery, Manchester Royal Infirmary, Oxford Rd, Manchester M13 9WL, UK.

Received 9 November 1984

Accepted 2 March 1985 well defined trigger spot. She had minimal nystagmus on looking to her right.

Computed tomography revealed an ectatic basilar artery, curving into the right cerebello-pontine angle. Ito was felt unjustifiable to perform vertebral angiography an $\mathbb{N}$ at that time we did not have the facility for intravenouß 응 digital subtraction angiography. Percutaneous ther $\vec{D}$. mocoagulation was performed with good relief of heô $\vec{c}$ pain. One year later, she returned with a recurrence of trigeminal neuralgia but she refused a further radiofre 8 quency lesion. Although her pain persists 14 months afte the recurrence, it is ameliorated with drug therapy.

\section{Case 2}

A Caucasian female, aged 82 years, complained of trigeminal neuralgia which had been present for 9 months. After an initial favourable response to carbamazepine, her pain returned and she underwent infraorbital alcohol injection at another hospital. Relief was minimal and she was referred to our department. The patient complained of left sided trigeminal neuralgia affecting the second and third divisions. She was normotensive and was generally well. A CT scan revealed an ectatic basilar artery occupying the left cerebello-pontine angle. Angiography was not performed. Percutaneous thermocoagulation relieved her pain but one year later her pain returned in the second division of her left trigeminal nerve territory. Radiofrequency thermocoagulation was repeated with good initial relief. She remains free of pain 14 months later.

Case 3

A 71-year-old Caucasian male presented with an 8 year history of trigeminal neuralgia affecting the first and second divisions of the left trigeminal territory. Initially, his pain had responded to carbamazepine but the patient was unable to tolerate this drug. He was mildly hypertensive and was receiving methyl dopa. Several years previously, he had had a cerebrovascular accident which left him with 
a mild left hemiparesis. Three years previously, he had sustained a myocardial infarction but, apart from his trigeminal neuralgia, he was well when he presented to us. The patient's pain had become unbearable. It was triggered by yawning, looking downwards and eased by lying on his left side. A CT scan revealed an ectatic basilar artery (fig 1a, b) and an intravenous digital subtraction angiogram (DIVA IGE, LVA isocentric orbiting angiographic unit with D F 3000 digital system) (fig 2a, b) confirmed the presence of an ectatic basilar artery extending to the apex of the petrous bone on the left. The patient was clearly at some risk if a major surgical procedure were to be considered. For several reasons (see Discussion), vascular decompression via a posterior fossa exploration was consi-
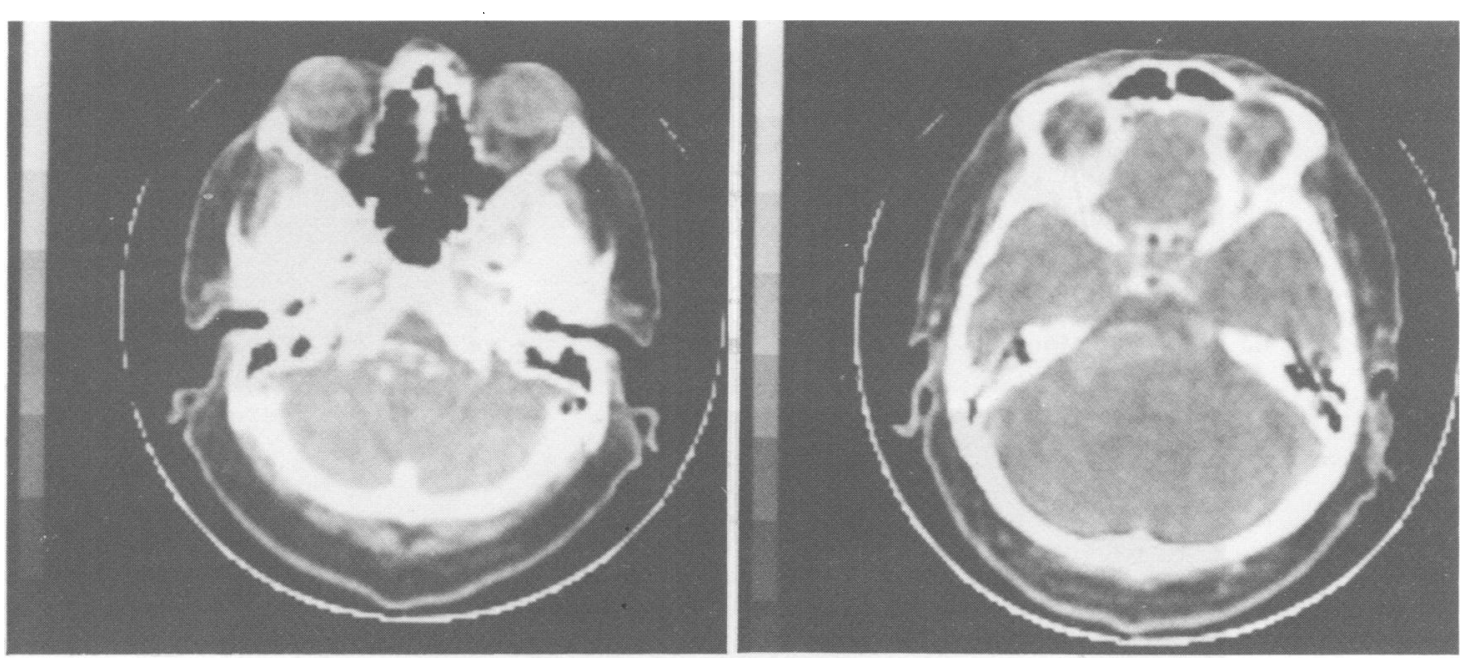

Fig 1 CT scan demonstrating ectatic basilar artery (Case 3) (a) before enhancement with intravenous contrast (b) after enhancement.
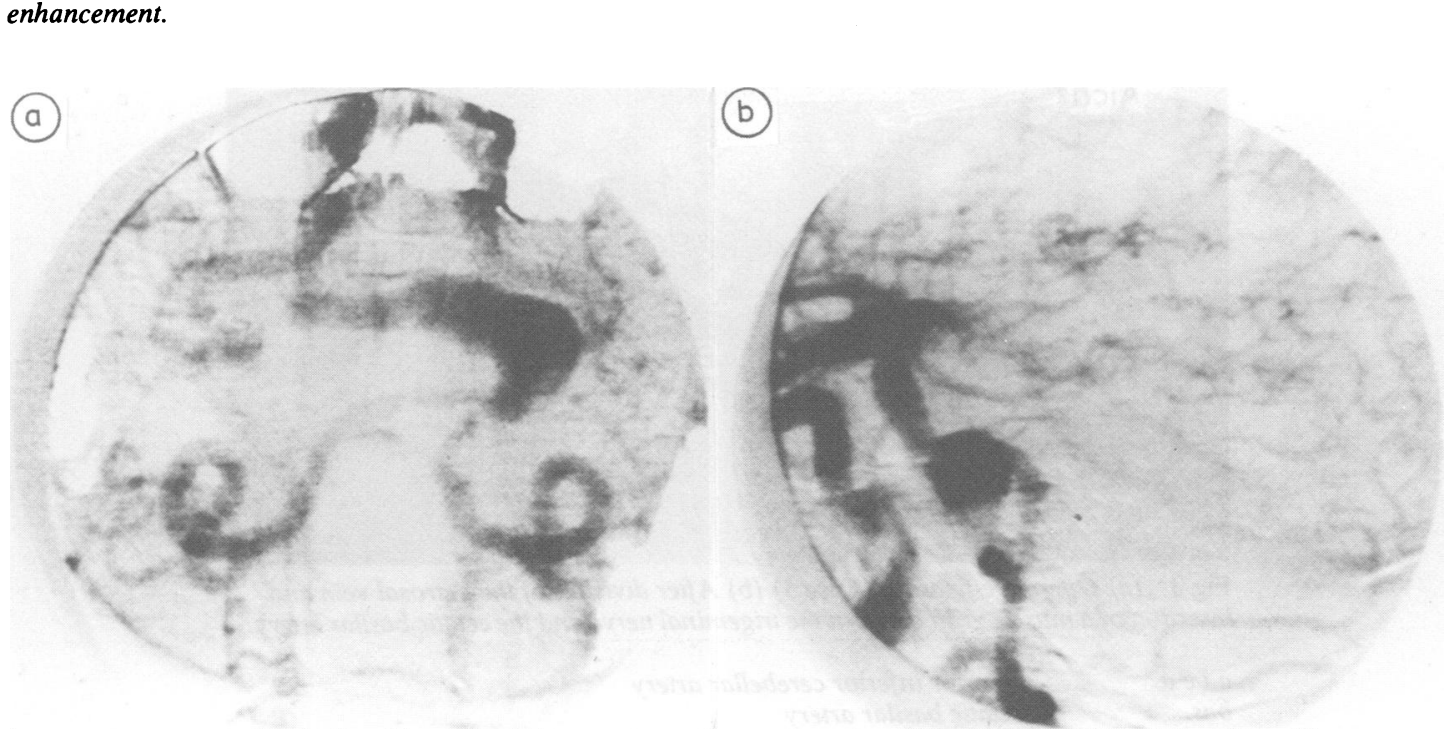

Fig 2 Intravenous digital subtraction angiogram demonstrating ectatic basilar artery (Case 3) (a) frontal (b) lateral view.

dered to be the operation of choice and after frank discussion with the patient, this was the procedure advised. At operation, a dilated, tortuous, atherosclerotic basilar artery was found lying antero-inferiorly to the trigeminal nerve. The petrosal vein crossed the nerve postero-superiorly, tethering the nerve on to the basilar artery by means of arachnoid adhesions (fig $3 \mathrm{a}$ ). The vein was divided and the nerve mobilised and delivered anterior to the artery. A muscle patch ${ }^{3}$ was then placed between nerve and artery (fig 3b). There were no changes in vital signs or ECG during the operation. After the operation, the patient was relieved of his trigeminal neuralgia. However, it was evident within 24 hours that he had suffered a myocardial infarction. Despite this, he made good progress until one 

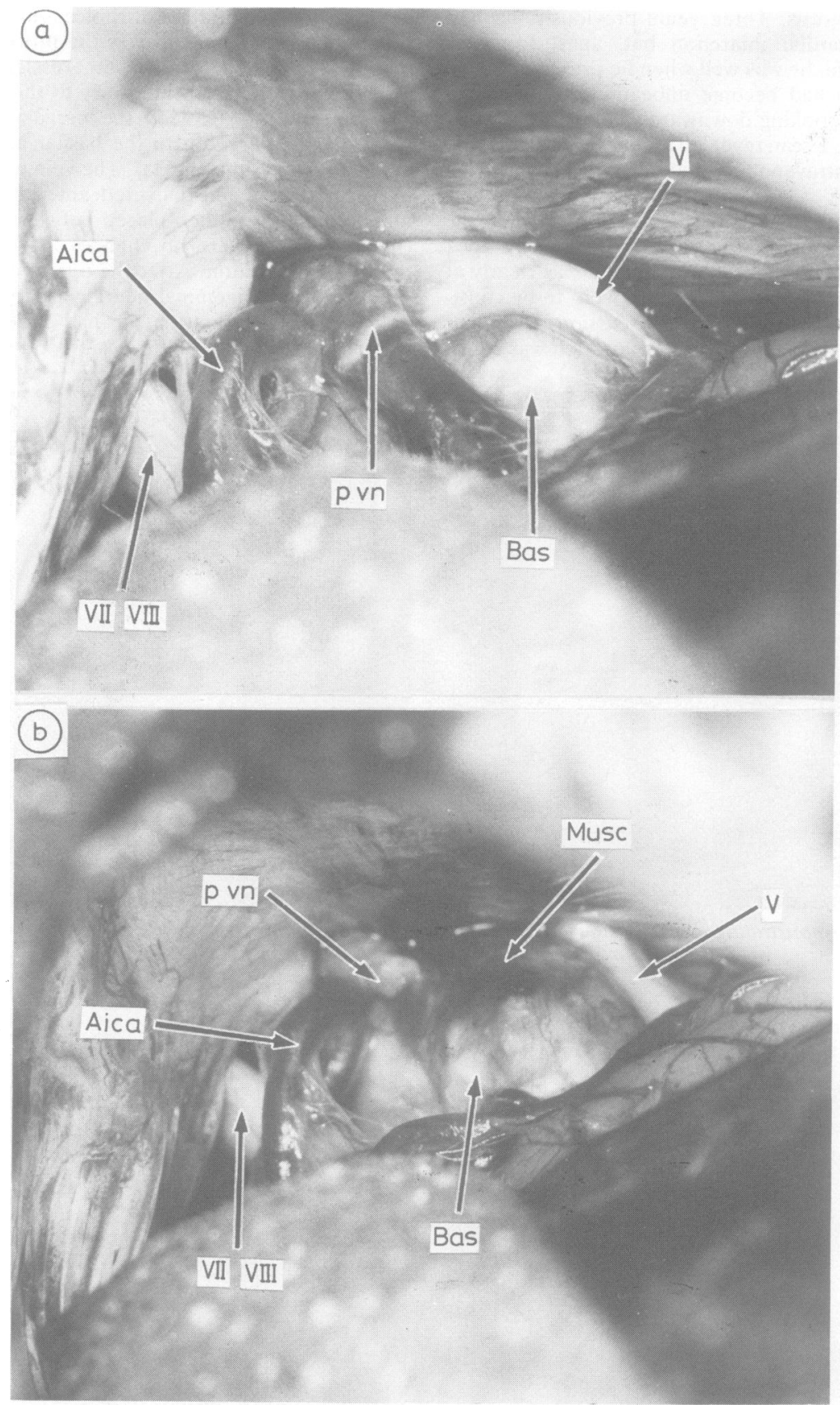

Fig 3 (a) Operative findings (Case 3) (b) After division of the petrosal vein and insertion of a muscle graft between the trigeminal nerve and the ectatic basilar artery.

$\begin{array}{ll}\text { a i c a } & \text { anterior inferior cerebellar artery } \\ \text { bas. } & \text { ectatic basilar artery } \\ \text { musc. } & \text { muscle graft } \\ p \text { vn. } & \text { petrosal vein } \\ V, V I I, V I I I & \text { cranial nerves. }\end{array}$


week after operation when he developed heart failure and subsequently non-ketotic hyperglycaemic hyperosmolar coma, from which he recovered. Unfortunately, he developed a chest infection complicated by septicaemia which was followed by a further myocardial infarction, intestinal infarction and ultimately he died. Post mortem examination revealed severe generalised atherosclerosis and confirmed the clinical diagnosis (fig 4). There was no evidence of brain stem infarction.

\section{Case 4}

A 59-year-old Caucasian female presented with a 5 year history of intractable trigeminal neuralgia affecting the second and third divisions of the right trigeminal territory. The pain was associated with an occasional left-sided tremor of the arm. Carbamazepine failed to afford relief. The patient was otherwise well apart from being mildly hypertensive (BP 170/100 $\mathrm{mm} \mathrm{Hg}$ ). A CT scan revealed an ectatic basilar artery (fig 5) with evidence of possible lacunar infarcts in both internal capsules. Subtraction angiography (fig 6a, b) confirmed the presence of an ectatic basilar artery extending into the right cerebello-pontine angle, subsequently crossing the midline to extend superiorly to the left as a fusiform dilatation. Posterior fossa exploration for vascular decompression of the trigeminal nerve was undertaken and a complex anatomical arrangement was observed (fig 7a). The anterior inferior cerebellar artery passed between the facial and auditory nerves before sending a labyrinthine branch to the internal

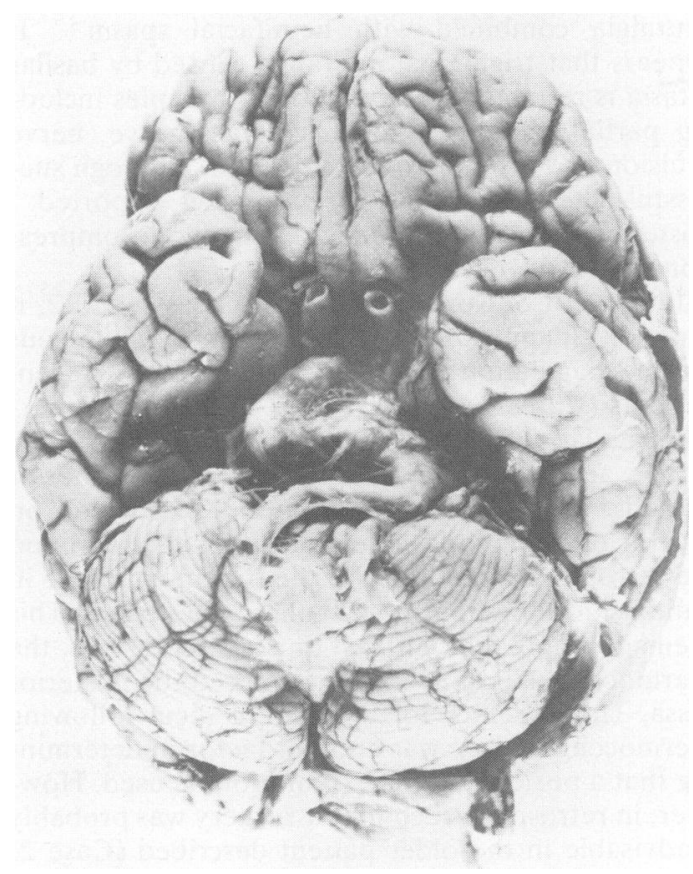

Fig 4 Post mortem specimen of brain (Case 3). Ventral aspect demonstrating ectatic basilar artery.

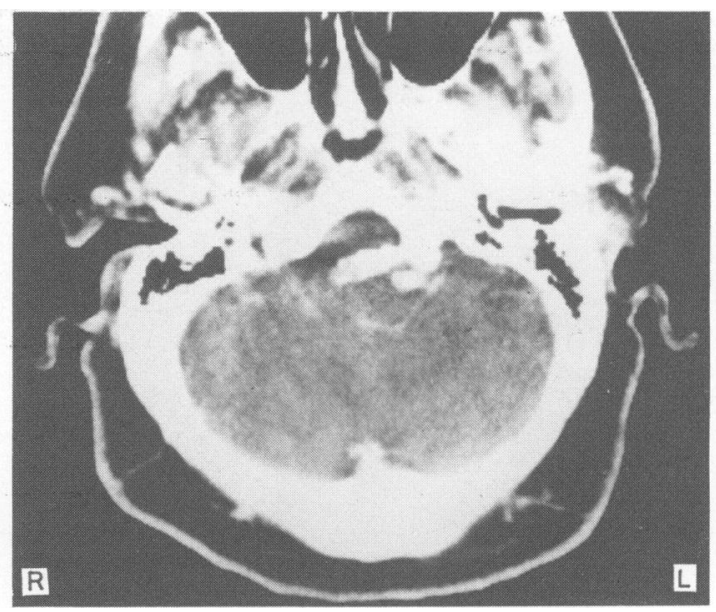

Fig 5 CT scan demonstrating ectatic basilar artery (Case 4). After enhancement with intravenous contrast.

auditory meatus. It is interesting that this patient had no evidence of hemifacial spasm, presumably because the artery was not in contact with the root entry zone ${ }^{y}$ of the facial nerve. The dilated atheromatous basilar artery passed deep to the facial and auditory nerves and deep to the anterior inferior cerebellar artery. The latter vessel was compressed against the root entry zone of the trigeminal nerve, indenting the nerve and elevating it. The petrosal vein ran below the trigeminal nerve just proximal to the site at which the nerve penetrated the dura and appeared to hitch the nerve to the under-side of the tentorium. Decompression of the trigeminal nerve involved three manoeuvres. Firstly, the anterior inferior cerebellar artery was mobilised and delivered from under the trigeminal nerve whilst preserving the labyrinthine artery. Secondly, a muscle graft was placed between the inferior surface of the nerve and the anterior inferior cerebellar artery and the basilar artery which was now in contact with the nerve. Thirdly, the petrosal vein was divided, so releasing the nerve from any tendency to be hitched up to the under-side of the tentorium (fig $7 \mathrm{~b}$ ). After operation, the patient had no neurological deficit and was free of pain. She had an episode of cerebrospinal rhinorrhoea, the source of which was an incompletely occluded mastoid air cell. She had minor repair to seal the mastoid air cells and was soon discharged home. She has remained free from her trigeminal neuralgia until the present time (14 months).

\section{Discussion}

Elongation and tortuosity of the basilar artery is variously referred to as: ectasia, ${ }^{10}$ arterectasis ${ }^{11}$ or cirsoid ("S" shaped) aneurysm of the basilar artery. ${ }^{8}$ The last term was used to denote "an " $S$ " shaped elongation of the basilar and/or vertebral arteries with consequent lateral bulging that compresses either the fifth or eighth nerve or both" ${ }^{8}$ The lateral 

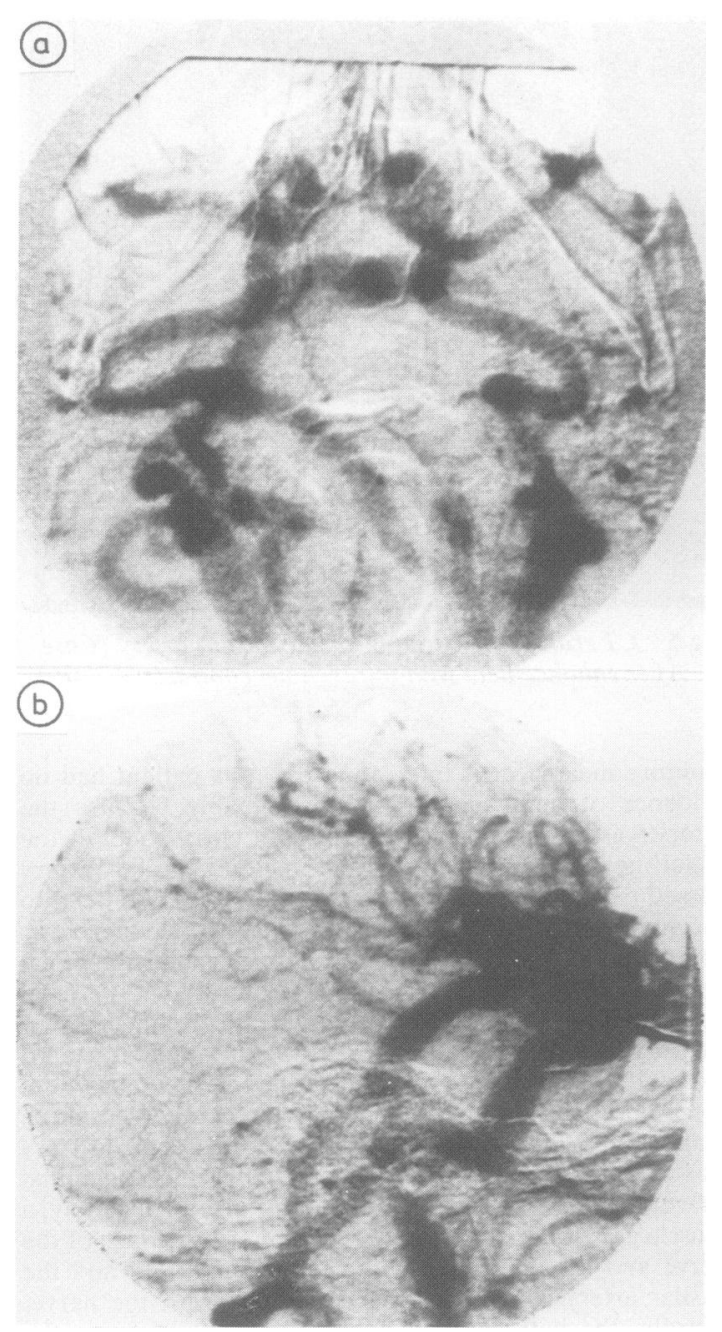

Fig 6 Intravenous digital subtraction angiogram demonstrating ectatic basilar artery (Case 4) (a) frontal, (b) lateral view.

bulging of the basilar artery is more often to the left than to the right. ${ }^{8}$ Ectasia is associated with hypertension and atherosclerosis ${ }^{12}{ }^{13}$ and was so with two of the four patients here reported. There is a high incidence of generalised vascular disease in patients with trigeminal neuralgia ${ }^{14}$ although this may be a reflection of the age group so afflicted with this complaint.

The incidence of basilar artery ectasia is difficult to assess. Sunderland reported two examples where the basilar artery was elongated and was found to impress the trigeminal nerve out of 210 necropsies. ${ }^{15}$ Dandy encountered 11 cases in 108 patients under- going posterior fossa exploration for treatment of trigeminal neuralgia. ${ }^{8}$ To some extent, the incidence of ectasia of the basilar artery in patients with trigeminal neuralgia will depend on the frequency with which such patients are investigated by angiography or posterior fossa exploration. The advent of CT scanning and more recently of intravenous digital subtraction angiography might be expected to increase the rate of detection since these investigations are less invasive and therefore more likely to be performed. In our department, of 112 consecutive patients with facial pain, 84 had CT scans with contrast enhancement. Atypical facial pain was present in six patients; multiple sclerosis was diagnosed in one patient and temporal arteritis in another. Of the remaining 76 patients with trigeminal neuralgia who had CT scans, basilar artery ectasia was detected in three (3.95\%); the fourth case of basilar artery ectasia described here was not included in this series of CT scans. This incidence is less than that reported by Dandy ${ }^{8}$ but his figures may, to some extent, reflect his case selection. Cases 3 and 4 emphasise the valuable information which may be provided by intravenous digital subtraction angiography examination now that this investigation is available.

Basilar artery ectasia has been described in association with trigeminal neuralgia ${ }^{8}$ and trigeminal neuralgia combined with hemifacial spasm. ${ }^{57}$ It appears that trigeminal neuralgia caused by basilar ectasia is refractory to a variety of therapies including partial section of the trigeminal nerve, nerve avulsion and foramen ovale injection, although successful thermocoagulation has been reported. ${ }^{10}$ Posterior fossa exploration and vascular decompression, however, has proved effective.?

In view of our experience with Cases 1 and 2, it was felt inappropriate to perform percutaneous thermocoagulation on two of our more recent patients (Cases 3 and 4). It was thought that thermocoagulation might damage the artery, since such ectatic arteries may rise even above the posterior clinoids. ${ }^{21316}$ Moreover, the author has noted on several occasions the arachnoid thickening around the retrogasserian portion of the trigeminal nerve in patients in whom thermocoagulation has failed. This seems to imply that, at least on some occasions, the thermocoagulation needle has entered the posterior fossa. The tendency for the pain to recur following thermocoagulation ${ }^{7}$ was another factor in determining that a posterior fossa exploration be used. However, in retrospect, such major surgery was probably inadvisable in the older patient described (Case 2) since the risks of thermocoagulation are usually less, although fatalities have occurred. ${ }^{17}$

The operative findings in Cases 3 and 4 emphasise 

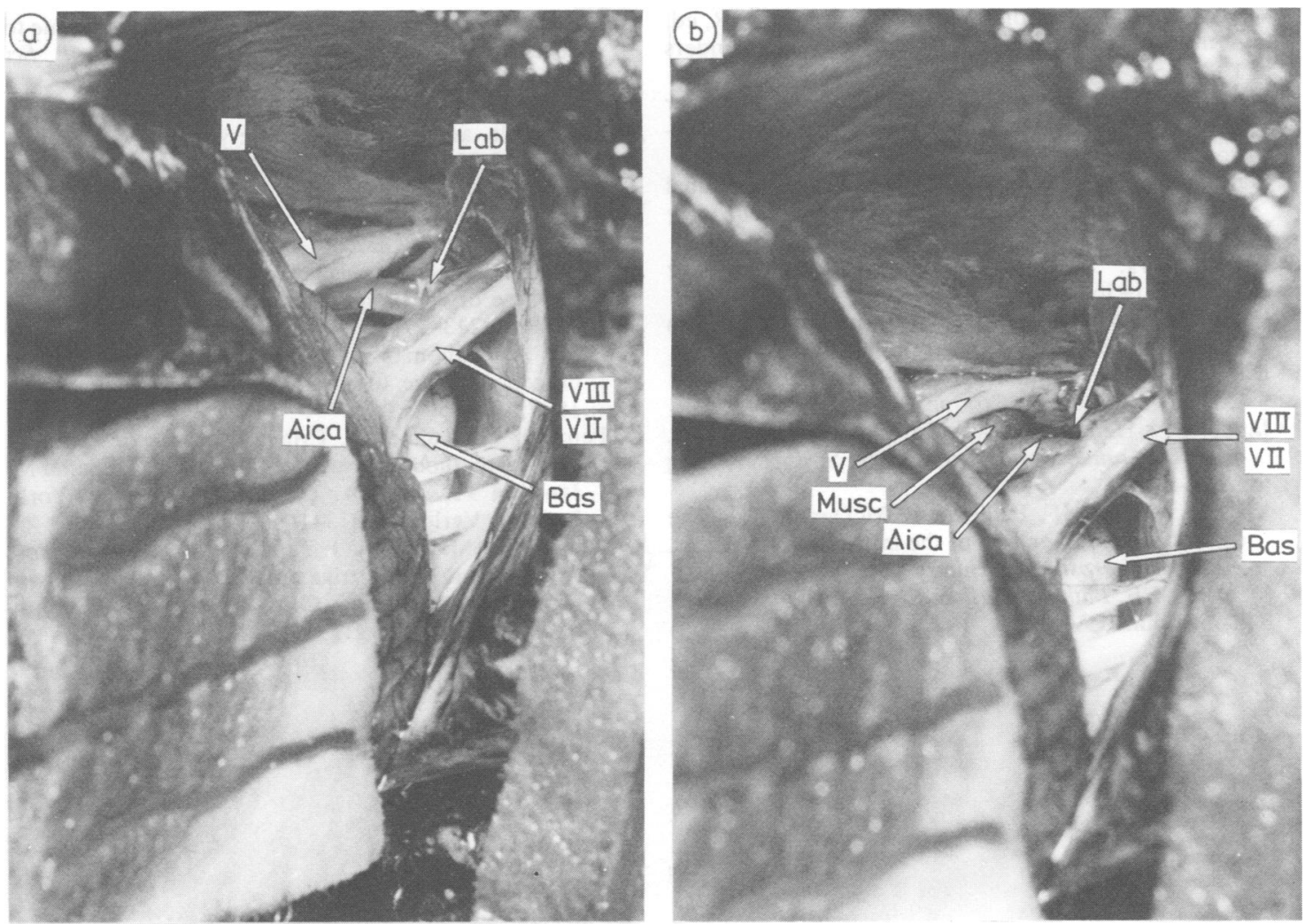

Fig 7 (a) Operative findings (Case 4) (b) After insertion of a muscle graft between the trigeminal nerve and arteries in contact with it. Note preservation of the labyrinthine artery.

$\begin{array}{ll}\text { a } i c \text { a } & \text { anterior inferior cerebellar artery } \\ \text { bas. } & \text { ectatic basilar artery } \\ \text { lab. } & \text { labyrinthine artery } \\ \text { musc. } & \text { muscle graft } \\ V, V I I, V I I I & \text { cranial nerves. }\end{array}$

the complex anatomical neuro-vascular relations which may occur in the posterior fossa. The possibility of vascular cross-compression as a cause of trigeminal neuralgia ${ }^{218}$ is clearly demonstrated by Case 4 where the anterior inferior cerebellar artery, basilar artery and petrosal vein all appeared to contribute to compression of the trigeminal nerve. The value of the operating microscope in providing good illumination and magnification, thus facilitating preservation of fine but important vessels such as the labyrinthine artery is obvious.

Clearly, caution must be exercised when advising microvascular decompression when thermocoagulation, although theoretically not without risk, might be safer. It should be remembered that patients with this type of vascular anomaly are likely to have generalised arterial disease and this may adversely affect the outcome of surgery. However, vascular decompression of the trigeminal nerve appears to be a logical and effective operation for the alleviation of trigeminal neuralgia caused by an ectatic basilar artery, especially in the younger and otherwise fit patient.

The author thanks Miss Pamela Brown for typing the manuscript and the Department of Medical Illustration, Manchester Royal Infirmary for help in preparing the illustrations.

\section{References}

' Dandy WE. Lesions of the cranial nerves, diagnosis and treatment. J Int Coll Surg 1939;2:5-14.

2 Jannetta PJ. Vascular decompression in trigeminal neuralgia. In: Samii M, Jannetta PJ, eds. The Cranial Nerves. New York: Springer Verlag 1981:331-40.

${ }^{3}$ Jannetta PJ. Treatment of trigeminal neuralgia by 
micro-operative decompression. In: Youmans JR, ed. Neurological Surgery. Philadelphia: WB Saunders 1982:3589-608.

${ }^{4}$ Rhoton AL. Microsurgical anatomy of the posterior fossa cranial nerves. Clin Neurosurg 1979;26:398462.

${ }^{5}$ Gardner WJ. Concerning the mechanism of trigeminal neuralgia and hemifacial spasm. J Neurosurg 1962;19:947-58.

${ }^{\circ}$ Gardner WJ. Trigeminal Neuralgia. Clin Neurosurg $1968 ; 15: 1-56$

' Campbell E, Keedy C. Hemifacial spasm: a note on etiology in two cases. J Neurosurg 1947;4:342-7.

${ }^{8}$ Dandy WE. Intracranial arterial aneurysms. Ithaca: Comstock Publishing Co. Inc. 1945:64-6.

' Jannetta PJ. Hemifacial spasm. In: Samii M, Jannetta PJ, eds. The Cranial Nerves. New York: Springer Verlag 1981:484-93.

${ }^{10}$ Yu YL, Moseley IF, Pullicino P, McDonald WI. The clinical picture of ectasia of the intracerebral arteries. $J$ Neurol Neurosurg Psychiatry 1982;45:29-36.

"Stebhens WE. Pathology of the Cerebral Blood Vessels. Saint Louis: CV Mosky, 1972.
12 Ekbom K, Greitz T, Kugelberg E. Hydrocephalus due to ectasia of the basilar artery. J Neurol Sci 1969;8:465-77.

${ }^{13}$ Greitz T, Löfstedt S. The relationship between the third ventricle and the basilar artery. Acta Radiologica 1954;42:85-100.

${ }^{14}$ Lew FH, Grant FC. Physiopathologic and pathoanatomic aspects of major trigeminal neuralgia. Arch Neurol Psychiatry 1938;40:1126-34.

15 Sunderland S. Neurovascular relations and anomalies at the base of the brain. J Neurol Neurosurg Psychiatry 1948; 11:243-57.

${ }^{16}$ Breig A, Ekbom K, Greitz T, Kugelberg E. Hydrocephalus due to elongated basilar artery. Lancet 1967;i:874-5.

17 Penzholz H, Kuhner A. Critical remarks on different surgical methods in trigeminal neuralgia. In: Samii $\mathbf{M}$, Jannetta PJ, eds. The Cranial Nerves. New York: Springer Verlag 1981:341-6.

${ }^{18}$ Haines SJ, Martinez AJ, Jannetta PJ. Arterial cross compression of the trigeminal nerve at the pons in trigeminal neuralgia. Case report with autopsy findings. J Neurosurg 1979;50:257-9. 